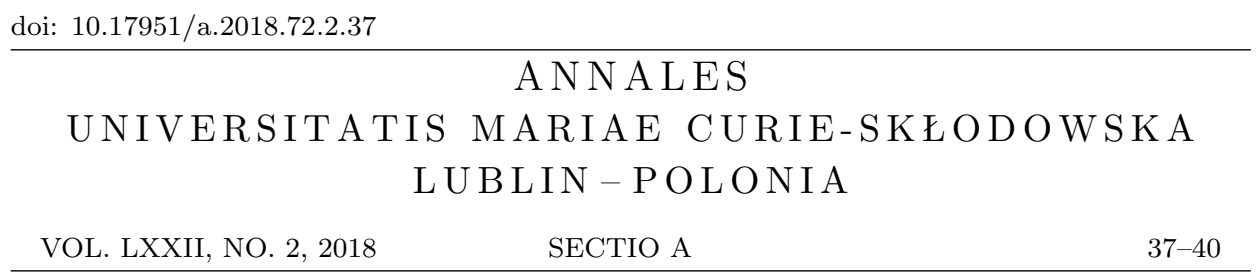

JAN KUREK and WŁODZIMIERZ M. MIKULSKI

\title{
On the existence of connections with a prescribed skew-symmetric Ricci tensor
}

\begin{abstract}
We study the so-called inverse problem. Namely, given a prescribed skew-symmetric Ricci tensor we find (locally) a respective linear connection.
\end{abstract}

1. Introduction. All manifolds and maps between manifolds considered in the paper are assumed to be smooth (i.e. of class $C^{\infty}$ ).

The concept of a linear connection $\nabla$ on a manifold $M$ and its Ricci tensor $S$ can be found in the fundamental monograph [4].

In the present paper, we study the so-called inverse problem.

More detailed, under some assumption on a tensor field $r$ of type $(0,2)$ on $M$, we prove the existence of a local solution of the equation

$$
S=r
$$

with unknown linear connection $\nabla$ on $M$.

In particular, we deduce that any 2 -form $\omega$ on a manifold $M$ with $\operatorname{dim}(M)$ $\geq 2$ is locally the Ricci tensor $S$ of some linear connection $\nabla$ on $M$.

In the analytic situation, the inverse problem was studied in many papers, e.g. [1, 2, 3, 5]. For example, in [5], using the Cauchy-Kowalevski theorem, the authors found (locally) all analytic linear connections for a prescribed analytic Ricci tensor. In the $C^{\infty}$ situation, we can not apply the CauchyKowalevski theorem.

2010 Mathematics Subject Classification. 53B05, 53C05.

Key words and phrases. Linear connection, Ricci tensor. 
From now on, $x^{1}, \ldots, x^{n}$ denote the usual coordinates on $\mathbf{R}^{n}$ and $\partial_{1}, \ldots, \partial_{n}$ denote the usual canonical vector fields on $\mathbf{R}^{n}$. Given a map $f: \mathbf{R}^{n} \rightarrow \mathbf{R}$ let $(f)_{i}:=\partial_{i}(f)=\frac{\partial f}{\partial x^{i}}$ for $i=1, \ldots, n$.

2. The main result. The main result of the paper is the following

Theorem 1. Let $M$ be a manifold such that $\operatorname{dim}(M) \geq 2$ and let $x_{o} \in$ $M$. Let $r$ be a tensor field of type $(0,2)$ on $M$ such that $r(X, X)=0$ around $x_{o}$ for some vector field $X \in \mathcal{X}(M)$ with $X_{x_{o}} \neq 0$. Then there is a linear connection $\nabla$ on $M$ such that $r$ is the Ricci tensor $S$ of $\nabla$ on some neighborhood of $x_{0}$.

Proof. We may assume that $M=\mathbf{R}^{n}, x_{o}=0$ and $X=\partial_{1}$.

Let $r$ be the tensor field of type $(0,2)$ on $\mathbf{R}^{n}$ and denote $r_{i j}=r\left(\partial_{i}, \partial_{j}\right)$ for $i, j=1, \ldots, n$. Then

$$
r_{11}=0 .
$$

The Ricci tensor $S$ of a linear connection $\nabla$ has the following rather well-known coordinate expression

(3) $S\left(\partial_{i}, \partial_{j}\right)=\sum_{k=1}^{n}\left[\left(\Gamma_{i j}^{k}\right)_{k}-\left(\Gamma_{k j}^{k}\right)_{i}\right]+\sum_{k, l=1}^{n}\left[\Gamma_{i j}^{l} \Gamma_{k l}^{k}-\Gamma_{k j}^{l} \Gamma_{i l}^{k}\right], i, j=1, \ldots, n$,

where $\Gamma_{j k}^{i}$ are the Christoffel symbols of $\nabla$, see [4].

It is sufficient to show that under assumption (2), equation (1) has a local solution (defined on some neighborhood of 0$) \nabla=\left(\Gamma_{b c}^{a}\right)$ such that

$$
\begin{aligned}
& \Gamma_{b c}^{a}=0 \text { for } a=3, \ldots, n, b, c=1, \ldots, n, \\
& \Gamma_{b c}^{2}=0 \text { for } b, c=2, \ldots, n, \\
& \Gamma_{b 1}^{2}=0 \text { for } b=1, \ldots, n, \\
& \Gamma_{1 b}^{1}=0 \text { for } b=1, \ldots, n .
\end{aligned}
$$

In other words, we put $\Gamma_{b c}^{a}=0$ for $a, b, c=1, \ldots, n$ except for $\Gamma_{1 j}^{2}$ with $j=2, \ldots, n$ and $\Gamma_{i j}^{1}$ with $i=2, \ldots, n$ and $j=1, \ldots, n$.

Using (4) and the coordinate expression (3), we get

(5) $S\left(\partial_{i}, \partial_{j}\right)=\sum_{k=1}^{2}\left(\Gamma_{i j}^{k}\right)_{k}-\sum_{\substack{k, l \in\{1,2\} \\ k \neq 1}} \Gamma_{k j}^{l} \Gamma_{i l}^{k}=\left(\Gamma_{i j}^{1}\right)_{1}+\left(\Gamma_{i j}^{2}\right)_{2}-\Gamma_{2 j}^{1} \Gamma_{i 1}^{2}-\Gamma_{1 j}^{2} \Gamma_{i 2}^{1}$ as $\Gamma_{b c}^{a}=0$ if $a=3, \ldots, n$ and $b, c=1, \ldots, n$, and $\Gamma_{a c}^{a}=0$ if $a, c=1, \ldots, n$.

Then using (5) and (4), we get

$$
\begin{gathered}
S\left(\partial_{1}, \partial_{1}\right)=0, \\
S\left(\partial_{1}, \partial_{j}\right)=\left(\Gamma_{1 j}^{2}\right)_{2} \text { for } j=2, \ldots, n,
\end{gathered}
$$




$$
\begin{gathered}
S\left(\partial_{i}, \partial_{1}\right)=\left(\Gamma_{i 1}^{1}\right)_{1} \text { for } i=2, \ldots, n, \\
S\left(\partial_{i}, \partial_{j}\right)=\left(\Gamma_{i j}^{1}\right)_{1}-\Gamma_{1 j}^{2} \Gamma_{i 2}^{1} \text { for } i, j=2, \ldots, n .
\end{gathered}
$$

More precisely, to obtain $(6)$ we use $(5)$ with $(i, j)=(1,1)$ and the assumed (in (4)) conditions $\Gamma_{11}^{1}=\Gamma_{11}^{2}=0$. To obtain (7), we use (5) with $(i, j)=(1, j)$ and the assumed (in (4)) conditions $\Gamma_{11}^{2}=\Gamma_{12}^{1}=\Gamma_{1 j}^{1}=0$. To obtain (8), we use (5) with $(i, j)=(i, 1)$ and the assumed (in (4)) conditions $\Gamma_{11}^{2}=\Gamma_{i 1}^{2}=0$. To obtain (9), we use (5) with $i, j=2, \ldots, n$ and the assumed (in (4)) conditions $\Gamma_{i 1}^{2}=\Gamma_{i j}^{2}=0$.

Then, by (2), (4) and (6)-(9), the equation (1) with unknown $\nabla$ satisfying (4) is equivalent to the system of systems of differential equations

$$
\begin{gathered}
\left(\Gamma_{1 j}^{2}\right)_{2}=r_{1 j} \text { for } j=2, \ldots, n, \\
\left(\Gamma_{i 1}^{1}\right)_{1}=r_{i 1} \text { for } i=2, \ldots, n, \\
\left(\Gamma_{i j}^{1}\right)_{1}=\Gamma_{1 j}^{2} \Gamma_{i 2}^{1}+r_{i j} \text { for } i, j=2, \ldots, n .
\end{gathered}
$$

It remains to observe that the system (10)-(12) has a solution of class $C^{\infty}$.

We see that the solution of (10) is

$$
\Gamma_{1 j}^{2}(x)=\int_{0}^{x^{2}} r_{1 j}\left(x^{1}, t, x^{3}, \ldots, x^{n}\right) d t+a_{j}\left(x^{1}, x^{3}, \ldots, x^{n}\right)
$$

for $j=2, \ldots, n$, and that the solution of (11) is

$$
\Gamma_{i 1}^{1}(x)=\int_{0}^{x^{1}} r_{i 1}\left(t, x^{2}, \ldots, x^{n}\right) d t+b_{i}\left(x^{2}, \ldots, x^{n}\right)
$$

for $i=2, \ldots, n$, where $a_{j}, b_{i}$ are arbitrary maps in $n-1$ variables.

Substituting the obtained $\Gamma_{1 j}^{2}$ into (12), we get the system of ordinary first order differential equations with parameters $x^{2}, \ldots, x^{n}$.

Such obtained system (12) has a solution of class $C^{\infty}$ according to the well-known theory of differential equations. We can even solve it explicitly as follows.

Each of the equations

$$
\left(\Gamma_{i 2}^{1}\right)_{1}=\Gamma_{12}^{2} \Gamma_{i 2}^{1}+r_{i 2} \text { for } i=2, \ldots, n
$$

(from the system (12)) is linear non-homogeneous with parameters. Solving them separately (using the well-known method), we obtain

$$
\begin{aligned}
\Gamma_{i 2}^{1}\left(x^{1}, \ldots,\right. & \left.x^{n}\right) \\
= & \left(\int_{0}^{x^{1}} r_{i 2}\left(t, x^{2}, \ldots, x^{n}\right) e^{-\int_{0}^{t} \Gamma_{12}^{2}\left(\tau, x^{2}, \ldots, x^{n}\right) d \tau} d t+c_{i 2}\left(x^{2}, \ldots, x^{n}\right)\right) \\
& \times e^{\int_{0}^{x^{1}} \Gamma_{12}^{2}\left(t, x^{2}, \ldots, x^{n}\right) d t}
\end{aligned}
$$


for $i=2, \ldots, n$, where $c_{i 2}$ are arbitrary maps in $n-1$ variables. Then the other equations of (12) (with $\Gamma_{i 2}^{1}$ as above) have solutions given by

$$
\begin{aligned}
\Gamma_{i j}^{1}\left(x^{1}, \ldots,\right. & \left.x^{n}\right) \\
= & \int_{0}^{x^{1}}\left(\Gamma_{1 j}^{2}\left(t, x^{2}, \ldots, x^{n}\right) \Gamma_{i 2}^{1}\left(t, x^{2}, \ldots, x^{n}\right)+r_{i j}\left(t, x^{2}, \ldots, x^{n}\right)\right) d t \\
& +d_{i j}\left(x^{2}, \ldots, x^{n}\right),
\end{aligned}
$$

where $d_{i j}$ are arbitrary maps in $n-1$ variables.

The proof of Theorem 1 is now complete.

We have the following interesting corollary of Theorem 1.

Corollary 1. Let $M$ be a manifold such that $\operatorname{dim}(M) \geq 2$ and let $x_{o} \in M$. Let $\omega$ be a 2-form on $M$. Then there is a linear connection $\nabla$ on $M$ such that $\omega$ is the Ricci tensor $S$ of $\nabla$ on some neighborhood of $x_{o}$.

Proof. For any vector field $X$ (in particular with $X_{x_{o}} \neq 0$ ) we have $\omega(X, X)$ $=0$. Then we apply Theorem 1 with $\omega$ playing the role of $r$.

\section{REFERENCES}

[1] Dušek, Z., Kowalski, O., How many are Ricci flat affine connections with arbitrary torsion?, Publ. Math. Debrecen 88 (3-4) (2016), 511-516.

[2] Gasqui, J., Connexions à courbure de Ricci donnée, Math. Z. 168 (2) (1979), 167-179.

[3] Gasqui, J., Sur la courbure de Ricci d'une connexion linéaire, C. R. Acad. Sci. Paris Sér A-B 281 (11) (1975), 389-391.

[4] Kobayashi, S., Nomizu, K., Foundation of Differential Geometry, Vol. I, J. WileyInterscience, New York, 1963.

[5] Opozda, B., Mikulski, W. M., The Cauchy-Kowalevski theorem applied for counting connections with a prescribed Ricci tensor, Turkish J. Math. 42 (2) (2018), 528-536.

Jan Kurek

Institute of Mathematics

Maria Curie-Skłodowska University

pl. M. Curie-Skłodowskiej 1

20-031 Lublin

Poland

e-mail: kurek@hektor .umcs.lublin.pl

Received October 3, 2018
Włodzimierz M. Mikulski

Institute of Mathematics

Jagiellonian University

ul. S. Łojasiewicza 6

30-348 Cracow

Poland

e-mail: Wlodzimierz.Mikulski@im.uj.edu.pl 